\title{
An Integral Mean Value Theorem concerning Two Continuous Functions and Its Stability
}

\author{
Monea Mihai \\ University Politehnica of Bucharest, Splaiul Independenţei 313, 060042 Bucharest, Romania \\ Correspondence should be addressed to Monea Mihai; mihaimonea@yahoo.com \\ Received 5 July 2015; Revised 12 September 2015; Accepted 15 September 2015 \\ Academic Editor: Yaozhong Hu
}

Copyright ( 2015 Monea Mihai. This is an open access article distributed under the Creative Commons Attribution License, which permits unrestricted use, distribution, and reproduction in any medium, provided the original work is properly cited.

The aim of this paper is to investigate an integral mean value theorem proposed by one of the references of this paper. Unfortunately, the proof contains a gap. First, we present a counterexample which shows that this theorem fails in this form. Then, we present two improved versions of this theorem. The stability of the mean point arising from the second result concludes this paper.

\section{Introduction}

The mean value theorems represent some of the most useful mathematical analysis tools. The first known result is due to Lagrange (1736-1813). In the years that followed, more mathematicians investigated this subject. As consequences of this fact, now we can find similar results, more generalizations, or extensions. Sahoo and Riedel's book [1] presents a large collection of old and new mean value theorems. The readers can consult [2], [3], [4], or [5] to find some recent results. Reference [6] leads this subject to a new direction.

In [7], the following theorem was presented.

Theorem 1. Let $f$ and $g$ be two nonnegative continuous functions on the interval $[a, b]$ and $\lambda \in(0,1)$. There exists $c \in(a, b)$ such that

$$
\begin{aligned}
\int_{a}^{b} f(x) g(x) d x= & \lambda f(c) \int_{a}^{b} g(x) d x \\
& +(1-\lambda) g(c) \int_{a}^{b} f(x) d x .
\end{aligned}
$$

This result is very generous. Moreover, the authors obtained some interesting consequences. Unfortunately, the proof contains a gap. The aim of this paper is to present a counterexample which shows that the result from the previous theorem is not necessarily valid under this hypothesis. Afterwards, we present some conditions for which equality (1) holds. Finally, we include a stability result.

\section{A Counterexample for Theorem 1}

Let us consider the functions $f, g:[0,1] \rightarrow \mathbb{R}$ defined, for any $x \in[0,1]$, by $f(x)=x$ and $g(x)=1-x$. These functions are continuous and nonnegative. We have

$$
\begin{aligned}
& \int_{0}^{1} f(x) d x=\left.\frac{x^{2}}{2}\right|_{0} ^{1}=\frac{1}{2}, \\
& \int_{0}^{1} g(x) d x=\left.x\right|_{0} ^{1}-\left.\frac{x^{2}}{2}\right|_{0} ^{1}=1-\frac{1}{2}=\frac{1}{2} .
\end{aligned}
$$

We choose $\lambda=1 / 2$. Then

$$
\begin{aligned}
\lambda f & (c) \int_{0}^{1} g(x) d x+(1-\lambda) g(c) \int_{0}^{1} f(x) d x \\
& =\frac{1}{4} c+\frac{1}{4}(1-c)=\frac{1}{4},
\end{aligned}
$$

for any $c \in(0,1)$. Hence

$$
\begin{aligned}
\int_{0}^{1} f(x) g(x) d x & =\int_{0}^{1}\left(x-x^{2}\right) d x=\left.\frac{x^{2}}{2}\right|_{0} ^{1}-\left.\frac{x^{3}}{3}\right|_{0} ^{1} \\
& =\frac{1}{2}-\frac{1}{3}=\frac{1}{6} .
\end{aligned}
$$

Then, relation (1) fails. 


\section{Two Integral Mean Value Theorems}

In this section we present two valid versions of Theorem 1, which were suggested by their original proofs.

Theorem 2. Let $f$ and $g$ be two nonnegative continuous functions on the interval $[a, b]$. One supposes that there exists $u, v \in$ $[a, b]$ such that $\max f=f(u), \max g=g(u), \min f=f(v)$, and $\min g=g(v)$. Then, for any $\lambda \in(0,1)$, there exists $c \in$ $(a, b)$ such that equality (1) holds.

Proof. First, for every $x \in[a, b]$, we have $f(v) \leq f(x) \leq f(u)$. Hence, $g$ is nonnegative; then

$$
f(v) g(x) \leq f(x) g(x) \leq f(u) g(x)
$$

and we obtain

$$
\begin{aligned}
f(v) \int_{a}^{b} g(x) d x & \leq \int_{a}^{b} f(x) g(x) d x \\
& \leq f(u) \int_{a}^{b} g(x) d x .
\end{aligned}
$$

Equality in any side of (6) holds if $f$ is constant or $g \equiv 0$. Similarly, we obtain

$$
\begin{aligned}
g(v) \int_{a}^{b} f(x) d x & \leq \int_{a}^{b} f(x) g(x) d x \\
& \leq g(u) \int_{a}^{b} f(x) d x .
\end{aligned}
$$

We obtain equality in any side of (7) if $f \equiv 0$ or $g$ is constant.

Let $\lambda \in(0,1)$. Let us consider the continuous function $h:[a, b] \rightarrow \mathbb{R}$ defined, for any $t \in[a, b]$, by

$$
\begin{aligned}
h(t)= & \lambda f(t) \int_{a}^{b} g(x) d x+(1-\lambda) g(t) \int_{a}^{b} f(x) d x \\
& -\int_{a}^{b} f(x) g(x) d x .
\end{aligned}
$$

Then

$$
\begin{aligned}
& h(u) \\
& =\lambda\left(f(u) \int_{a}^{b} g(x) d x-\int_{a}^{b} f(x) g(x) d x\right) \\
& \quad+(1-\lambda)\left(g(u) \int_{a}^{b} f(x) d x-\int_{a}^{b} f(x) g(x) d x\right) .
\end{aligned}
$$

From (6) and (7), we obtain $h(u) \geq 0$. In the same mode, we have $h(v) \leq 0$. Then, there exists $c$ between $u$ and $v$ such that $h(c)=0$. We obtain (1).

It remains to show that $c \in(a, b)$. If $c \notin\{u, v\}$, then the conclusion is clear. We suppose that $c=u$. Using (6) and (7), we conclude that $f$ and $g$ are constant functions. We obtain $h(t)=0$, for any $t \in[a, b]$. Then, we can replace $c$ with any point from $(a, b)$. A similar conclusion is obtained if $c=v$. Now, the proof is complete.
As consequences of the previous theorem, we obtain the following result.

Theorem 3. Let $f$ and $g$ be two nonnegative continuous functions on the interval $[a, b]$ and monotone of the same type. Let $\lambda \in(0,1)$. Then there exists $c \in(a, b)$ such that equality (1) holds.

Proof. We suppose that $f$ and $g$ are increasing functions. We have $\max f=f(b), \max g=g(b)$, $\min f=f(a)$, and $\min g=g(a)$. The conclusion follows by applying the previous theorem.

\section{A Stability Result}

The parents of the stability concept are considered to be the mathematicians Ulam and Hyers (see [8-10]). This notion is associated with the functional equations, the differential equations, or the linear recurrences. Starting with [11], a new direction was created. It is about the stability of the point arising from the mean value theorems. Reference [12] or [13] is relevant.

We want to conclude this paper with a new stability result. It is about the Hyers-Ulam stability of the mean point defined by Theorem 3 . We apply a similar reasoning to Theorem 1 from [13].

Theorem 4. Let $\lambda \in(0,1)$. Let $f$ and $g$ be two nonnegative continuous functions on the interval $[a, b]$ and monotone of the same type. One assumes that there exists a unique $c \in(a, b)$ which is satisfying equality (1). Then, for any $\varepsilon>0$, there exists $\delta>0$ with the following property: for any continuous functions $F, G:[a, b] \rightarrow \mathbb{R}$ such that $|F(x)-f(x)|<\delta$ and $\mid G(x)-$ $g(x) \mid<\delta$, for any $x \in[a, b]$, there exists $d \in(a, d)$ such that $|d-c|<\varepsilon$ and

$$
\begin{aligned}
\int_{a}^{b} F(x) G(x) d x= & \lambda F(d) \int_{a}^{b} G(x) d x \\
& +(1-\lambda) G(d) \int_{a}^{b} F(x) d x
\end{aligned}
$$

Proof. We assume that the functions $f$ and $g$ are increasing. We define the function $h:[a, b] \rightarrow \mathbb{R}$ by

$$
\begin{aligned}
h(t)= & \lambda f(t) \int_{a}^{b} g(x) d x+(1-\lambda) g(t) \int_{a}^{b} f(x) d x \\
& -\int_{a}^{b} f(x) g(x) d x,
\end{aligned}
$$

for any $t \in[a, b]$. We have $h(c)=0$. Moreover,

$$
\begin{aligned}
h(a)= & \lambda f(a) \int_{a}^{b} g(x) d x+(1-\lambda) g(a) \int_{a}^{b} f(x) d x \\
& -\int_{a}^{b} f(x) g(x) d x
\end{aligned}
$$




$$
\begin{aligned}
= & \lambda \int_{a}^{b}(f(a)-f(x)) g(x) d x \\
& +(1-\lambda) \int_{a}^{b} f(x)(g(a)-g(x)) d x \leq 0 .
\end{aligned}
$$

The uniqueness of $c$ goes to $h(a)<0$. Similar arguments show that $h(b)>0$. Then $h(x)<0$, for any $x \in[a, c)$, and $h(x)>0$, for any $x \in(c, b]$.

Now, let $\delta>0$. For any continuous functions $F, G$ : $[a, b] \rightarrow \mathbb{R}$ such that $|F(x)-f(x)|<\delta$ and $|G(x)-g(x)|<\delta$, for any $x \in[a, b]$, we define the function $H:[a, b] \rightarrow \mathbb{R}$ by

$$
\begin{aligned}
H(t)= & \lambda F(t) \int_{a}^{b} G(x) d x+(1-\lambda) G(t) \int_{a}^{b} F(x) d x \\
& -\int_{a}^{b} F(x) G(x) d x,
\end{aligned}
$$

for any $t \in[a, b]$. Further,

$$
\begin{aligned}
& \left|F(t) \int_{a}^{b} G(x) d x-f(t) \int_{a}^{b} g(x) d x\right| \\
& \quad=\mid F(t) \int_{a}^{b} G(x) d x-f(t) \int_{a}^{b} G(x) d x \\
& \quad+f(t) \int_{a}^{b} G(x) d x-f(t) \int_{a}^{b} g(x) d x|\leq| F(t) \\
& \quad-f(t)\left|\int_{a}^{b} G(x) d x+\right| f(t)\left|\int_{a}^{b}\right| G(x)-g(x) \mid d x \\
& \quad \leq \delta\left(\int_{a}^{b}|G(x)-g(x)| d x+\int_{a}^{b} g(x) d x\right)+f(b) \\
& \quad \cdot \delta(b-a) \leq \delta^{2}(b-a)+\delta g(b)(b-a)+f(b) \delta(b \\
& \quad-a)
\end{aligned}
$$

and we obtain

$$
\begin{aligned}
& \left|F(t) \int_{a}^{b} G(x) d x-f(t) \int_{a}^{b} g(x) d x\right| \\
& \leq \delta^{2}(b-a)+\delta(g(b)+f(b))(b-a),
\end{aligned}
$$

for any $t \in[a, b]$. In the same mode, we obtain

$$
\begin{aligned}
& \left|G(t) \int_{a}^{b} F(x) d x-g(t) \int_{a}^{b} f(x) d x\right| \\
& \leq \delta^{2}(b-a)+\delta(g(b)+f(b))(b-a),
\end{aligned}
$$

for any $t \in[a, b]$. Moreover, we have

$$
\begin{aligned}
& \left|\int_{a}^{b} F(x) G(x) d x-\int_{a}^{b} f(x) g(x) d x\right| \\
& \leq\left|\int_{a}^{b} F(x) G(x) d x-\int_{a}^{b} f(x) G(x) d x\right| \\
& \quad+\left|\int_{a}^{b} f(x) G(x) d x-\int_{a}^{b} f(x) g(x) d x\right| \\
& \leq \delta \int_{a}^{b}|G(x)| d x+\delta \int_{a}^{b} f(x) d x \\
& \leq \delta \int_{a}^{b}|G(x)-g(x)| d x+\delta \int_{a}^{b} g(x) d x \\
& \quad+\delta \int_{a}^{b}|f(x)| d x \\
& \leq \delta^{2}(b-a)+\delta g(b)(b-a)+\delta f(b)(b-a),
\end{aligned}
$$

so

$$
\begin{aligned}
& \left|\int_{a}^{b} F(x) G(x) d x-\int_{a}^{b} f(x) g(x) d x\right| \\
& \quad \leq \delta^{2}(b-a)+\delta \cdot(g(b)+f(b))(b-a) .
\end{aligned}
$$

By using (15), (16), and (18), we obtain

$$
\begin{aligned}
& |H(t)-h(t)| \\
& \leq \lambda\left|F(t) \int_{a}^{b} G(x) d x-f(t) \int_{a}^{b} g(x) d x\right| \\
& \quad+(1-\lambda)\left|G(t) \int_{a}^{b} F(x) d x-g(t) \int_{a}^{b} f(x) d x\right| \\
& \quad+\left|\int_{a}^{b} F(x) G(x) d x-\int_{a}^{b} f(x) g(x) d x\right| \\
& \leq 2 \delta^{2}(b-a)+2 \delta(g(b)+f(b))(b-a),
\end{aligned}
$$

for any $t \in[a, b]$.

Now, let $\varepsilon>0$ and $p \in[a, c)$ such that $|c-p|<\varepsilon$. Similarly, let $q \in(c, b]$ such that $|c-q|<\varepsilon$. Then $h(p)<0<h(q)$. We consider $v>0$ such that

$$
h(p)<h(p)+v<0<h(q)-v<h(q) .
$$

We consider $\delta>0$ such that

$$
2 \delta^{2}(b-a)+2 \delta(g(b)+f(b))(b-a)<\nu .
$$

Further, $|H(p)-h(q)|<v$, so $H(p)<0$. Similarly, we have $|H(q)-h(q)|<v$, so $H(q)>0$. Then, there exists $d \in(p, q)$ such that $H(d)=0$. From $d \in(p, q)$, we obtain $|d-c|<\varepsilon$. The equality $H(d)=0$ goes to

$$
\begin{aligned}
\int_{a}^{b} F(x) G(x) d x= & \lambda F(d) \int_{a}^{b} G(x) d x \\
& +(1-\lambda) G(d) \int_{a}^{b} F(x) d x
\end{aligned}
$$

and the proof is complete. 


\section{Conflict of Interests}

The author declares that there is no conflict of interests regarding the publication of this paper.

\section{Acknowledgment}

This work has been funded by the Sectoral Operational Programme Human Resources Development 2007-2013 of the Ministry of European Funds through the Financial Agreement POSDRU 187/1.5/S/155420.

\section{References}

[1] P. K. Sahoo and T. Riedel, Mean Value Theorems and Functional Equations, World Scientific Publishing Company, New Jersey, NJ, USA, 1998.

[2] J. Molnárová, "On generalized Flett's mean value theorem," International Journal of Mathematics and Mathematical Sciences, vol. 2012, Article ID 574634, 7 pages, 2012.

[3] J. Tong, "The mean value theorem of Lagrange generalised to involve two functions," The Mathematical Gazette, vol. 84, no. 501, pp. 515-516, 2000.

[4] J. Tong, "A generalization of the mean value theorem for integrals," The College Mathematics Journal, vol. 33, no. 5, pp. 408-409, 2002.

[5] E. Wachnicki, "Une variante du théorème de Cauchy de la valeur moyenne," Demonstratio Mathematica, vol. 33, no. 4, pp. 737-740, 2000.

[6] R. C. Powers, T. Riedel, and P. K. Sahoo, "Flett's mean value theorem in topological vector spaces," International Journal of Mathematics and Mathematical Sciences, vol. 27, no. 11, pp. 689694, 2001.

[7] P. Khalili and D. Vasiliu, "An extension of the mean value theorem for integrals," International Journal of Mathematical Education in Science and Technology, vol. 41, no. 5, pp. 707-710, 2010.

[8] S. M. Ulam, A Collection of Mathematical Problems, Interscience, New York, NY, USA, 1960.

[9] S. M. Ulam, Problems in Modern Mathematics, Science Editions, Wiley, 1964.

[10] D. H. Hyers, "On the stability of the linear functional equation," Proceedings of the National Academy of Sciences of the United States of America, vol. 27, pp. 222-224, 1941.

[11] M. Das, T. Riedel, and P. K. Sahoo, "Hyers-Ulam stability of Flett's points," Applied Mathematics Letters, vol. 16, no. 3, pp. 269-271, 2003.

[12] P. Găvruţa, S.-M. Jung, and Y. Li, "Hyers-ULam stability of mean value points," Annals of Functional Analysis, vol. 1, no. 2, pp. 68-74, 2010.

[13] C. Mortici, M. Monea, and D. Ş. Marinescu, "The stability of some points arising from continuous, differential and integral expressions," Monatshefte für Mathematik, 2015. 


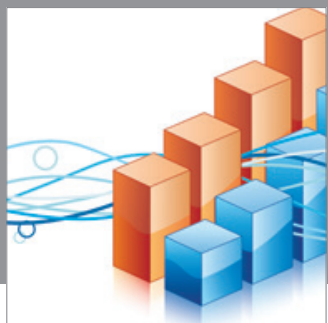

Advances in

Operations Research

mansans

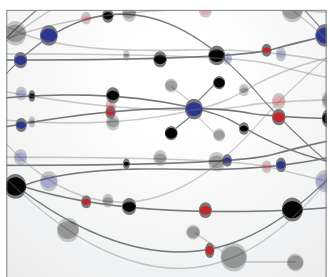

The Scientific World Journal
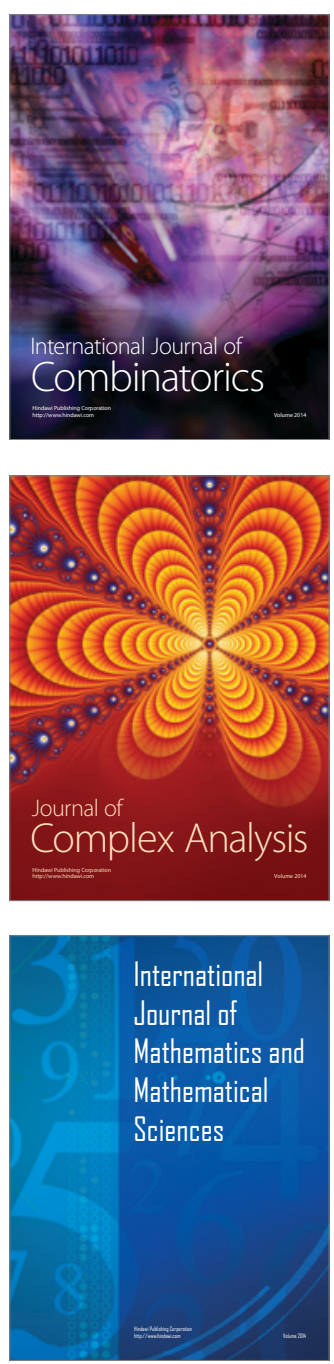
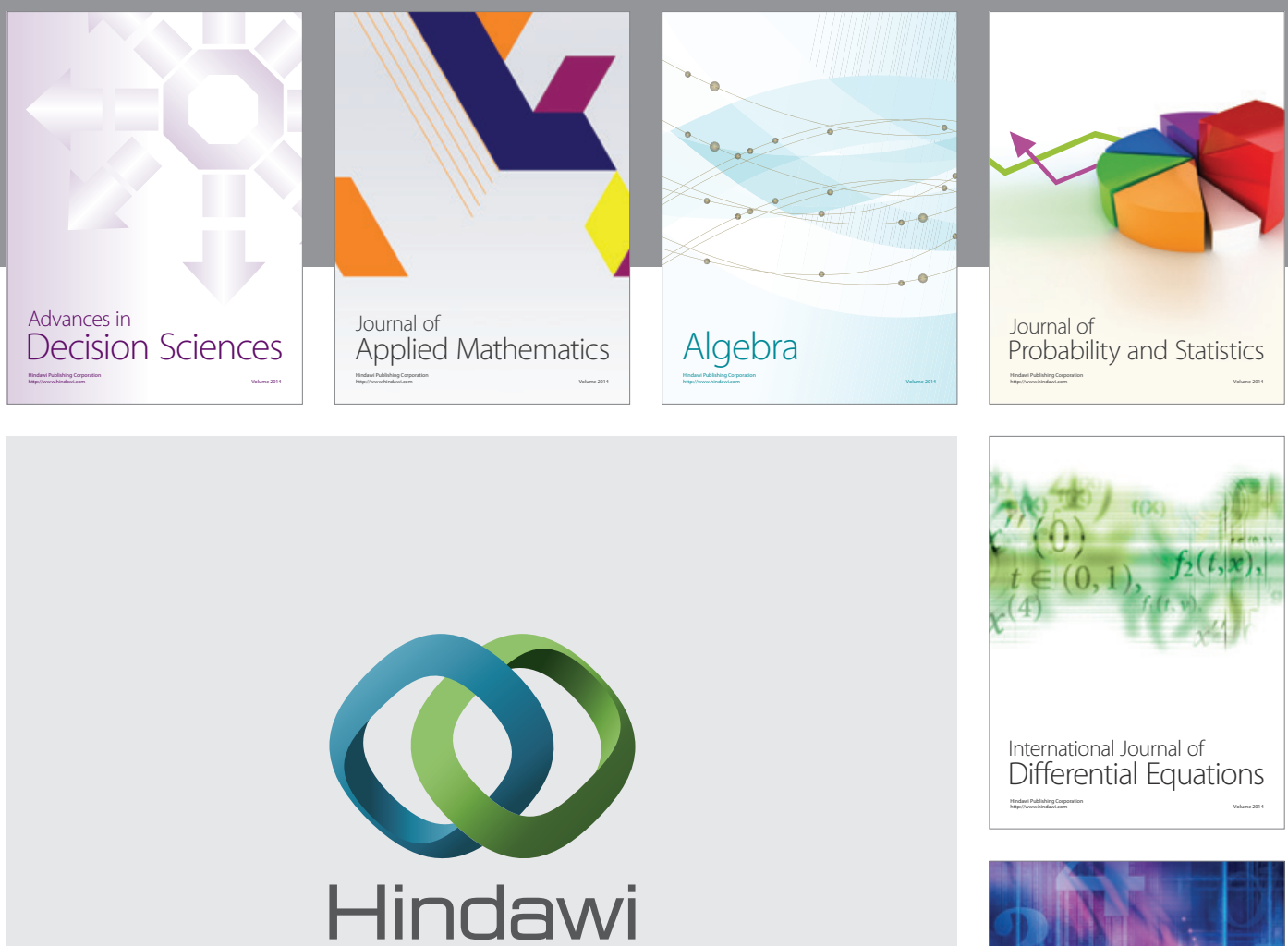

Submit your manuscripts at http://www.hindawi.com
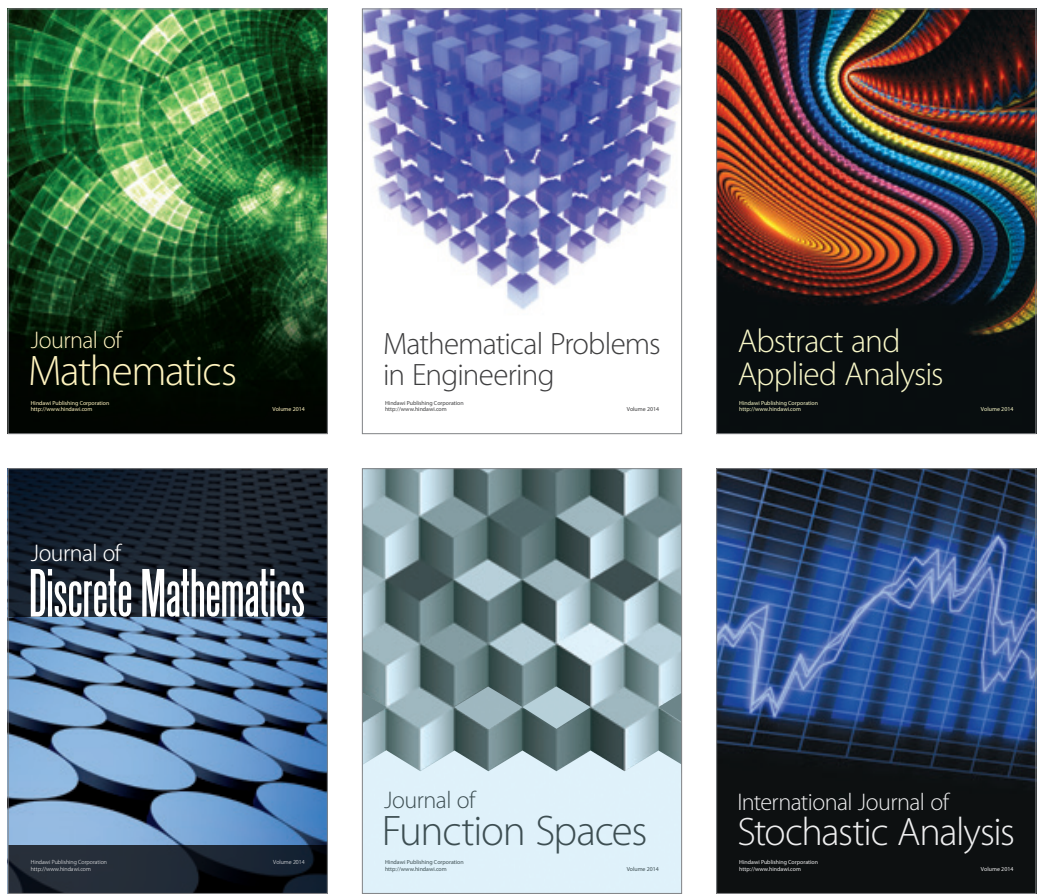

Journal of

Function Spaces

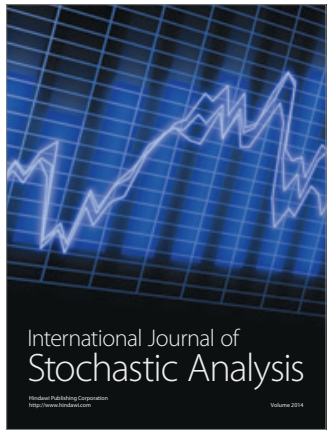

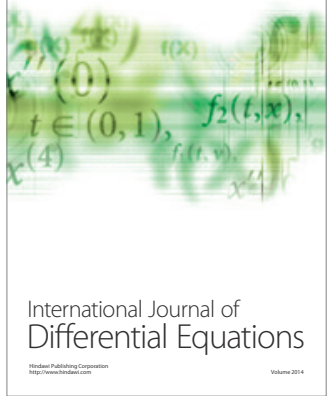
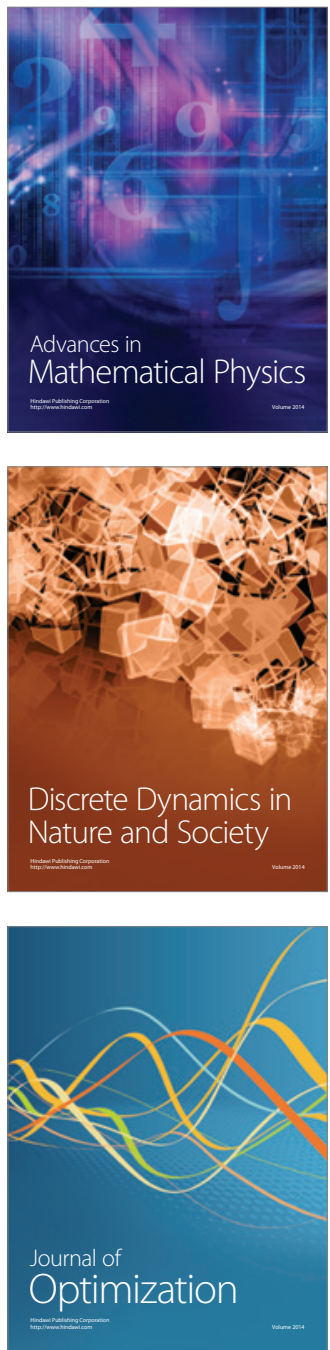